\section{ce \\ ...the \\ mechanism by \\ which loss of \\ AGL promotes \\ tumour growth \\ in bladder}

cancer...

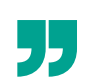

$\Rightarrow B L A D D E R$ CANCER

\title{
In the driving seat - AGL loss drives tumour growth
}

New data, published in Clinical Cancer Research, has shed light on the mechanism by which glycogen debranching enzyme (amylo- $\alpha-1,6-$ glucosidase, 4 - $\alpha$-gluganotransferase $(\mathrm{AGL})$ ) promotes tumour growth in bladder cancer.

Previous work by Guin and colleagues had identified AGL as a tumour growth suppressor and a prognostic marker, with low AGL expression predicting poor patient outcomes. The group's recent work sought to identify possible therapeutic targets or pathways that could be used to treat patients with low-AGL-expressing bladder tumours.

In vitro, UMUC3 human bladder cancer cells treated with short hairpin RNA (shRNA) specific for $A G L$ showed upregulation of 38 genes, seven of which had statistically significant positive correlation with at least three of four clinicopathological variables in human tumours. One of these genes, HAS2, is responsible for hyaluronic acid synthesis and could be a therapeutic target, so the group investigated whether hyaluronic acid synthesis and signalling are upregulated with AGL loss.

Short interfering RNA (siRNA) knock down of HAS2 in conjunction with shRNA knock down of AGL resulted in a marked reduction in UMUC3 proliferation and decreased anchorage-independent growth of these cells and also T24T cells, which have stable AGL loss. In high-HAS2expressing MGHU4 bladder cancer cells, depletion of HAS2 using siRNA also reduced proliferation. In two immortalized, nontransformed human urothelial cell lines, AGL depletion increased HAS2 expression and, in vivo, the bladders of $A G L$ knockout mice also showed increased HAS2 expression.

Inhibiting hyaluronic acid synthesis in low-AGL-expressing cells with 4-methylumbelliferone (4MU) reduced proliferation to a similar degree to that observed by knock down of HAS2. Cell-line-derived xenografts, in which HAS2 and $A G L$ had been knocked down, showed reduced tumour growth compared with xenografts with only AGL loss. Inhibition of hyaluronic acid synthesis in mice with AGL-depleted xenografts inhibited tumour growth.

In human bladder cancer, high HAS2 expression has been observed in high-grade and muscleinvasive tumours compared with low-grade non-muscle-invasive disease. In five independent patient datasets, $A G L$ expression negatively correlated with HAS2 expression, obtaining statistically significance in four. High HAS2 expression was associated with poor overall survival $(P=0.036)$, whereas patients with high $A G L$ expression in their bladder tumours had improved overall survival outcomes. Using combined $A G L$ and HAS2 expression data to stratify

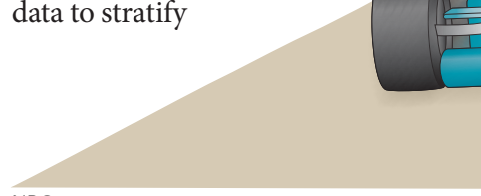

NPG patients, Guin and co-workers found that a patients with high AGL:HAS2 ratios had better overall survival $(P=0.001)$.

These data provide insight into the mechanism by which loss of AGL promotes tumour growth in bladder cancer via upregulation of HAS2 and increased hyaluronic acid synthesis. They give a rationale for personalized targeting of this signalling pathway in patients with low-AGL-expressing tumours using hyaluronic inhibitors such as $4 \mathrm{MU}$.

Louise Stone

ORIGINAL ARTICLE Guin, S. et al. Loss of glycogen debranching enzyme AGL drives bladder tumor growth via induction of hyaluronic acid synthesis. Clin. Cancer Res. http://dx.doi.org/ 10.1158/1078-0432.CCR-15-1706

FURTHER READING Ritterson Lew, C. et al. Targeting glycogen metabolism in bladder cancer. Nat. Rev. Urol. 12, 383-391 (2015) 